\title{
A New Strategy for Improving the Surface Quality of Ti6Al4V Machined by Abrasive Water Jet: Reverse Cutting with Variable Standoff Distances
}

\author{
Jie Xiong \\ Wuhan University \\ Liang Wan \\ Wuhan University \\ Yi'nan Qian \\ Wuhan University \\ Shuo Sun \\ Wuhan University \\ Deng Li ( $\sim$ 2008lee@whu.edu.cn ) \\ Wuhan University \\ Shijing Wu \\ Wuhan University
}

\section{Research Article}

Keywords: Abrasive water jet, Titanium alloys, Surface quality, Reverse cutting, Standoff distance

Posted Date: December 2nd, 2021

DOI: https://doi.org/10.21203/rs.3.rs-1107742/v1

License: (1) This work is licensed under a Creative Commons Attribution 4.0 International License. Read Full License

Version of Record: A version of this preprint was published at The International Journal of Advanced Manufacturing Technology on March 29th, 2022. See the published version at https://doi.org/10.1007/s00170-022-09091-6. 


\title{
A new strategy for improving the surface quality of Ti6Al4V machined by abrasive water jet: reverse cutting with variable standoff distances
}

\author{
Jie Xiong, Liang Wan, Yi’nan Qian, Shuo Sun, Deng Li*, Shijing Wu \\ 1. Hubei Key Laboratory of Waterjet Theory and New Technology, Wuhan University, Wuhan, 430072, China \\ 2. School of Power and Mechanical Engineering, Wuhan University, Wuhan, 430072, China \\ Corresponding author: Deng Li, E-mail:2008lee@whu.edu.cn
}

\begin{abstract}
Titanium alloys are widely used in important structures of aerospace vehicles, but the low thermal conductivity and high chemical activity make them difficult to process. As an untraditional machining technology, abrasive water jet (AWJ) has been proven to be an effective method for this kind of material. Aimed at further improving the cutting performance, reverse cutting with variable standoff distance (SOD) strategy was put forward, and experiments of titanium alloy Ti6Al4V machined by AWJ were conducted. The influence of SOD with different reverse cutting types on the kerf quality was studied to obtain the optimal SOD combinations. $R a, S a$ and kerf taper were used to evaluate the quality of the machined surface. Moreover, the results of reverse cutting at the same speed and efficiency and single cutting at the constant SOD were compared and analyzed. It was found that the proposed strategy results in higher kerf quality in the aspect of surface roughness, compared to the single cutting. To be more specific, for the reverse trimming cutting, the improvements of $R a$ and $S a$ can reach up to $62.8 \%$ and $73.1 \%$ respectively under the condition of the SOD of the second cutting is $8 \mathrm{~mm}$. Furthermore, the kerf taper can be reduced $26.1 \%$ when the SOD of the second cutting is $2 \mathrm{~mm}$. With respect to the reverse deepening cutting, even the traverse speed of reverse cutting is set as twice as that of a single cutting, the kerf quality is still better. Additionally, when the SOD of the second cutting is $4 \mathrm{~mm}$, the improvements of $R a$ and $S a$ can reach up to $51.7 \%$ and $14.9 \%$, respectively, and the kerf taper is reduced by $20.2 \%$. This study provides a new method for improving the machined surface quality of hard materials, especially for Ti6Al4V.
\end{abstract}

Keywords: Abrasive water jet, Titanium alloys, Surface quality, Reverse cutting, Standoff distance

\section{Introduction}

Titanium alloys (and more especially Ti6A14V) are widely used in the aerospace industry for the excellent physical properties of strength to weight ratio, high thermal and corrosion resistance, and chemical inertness [1]. However, Titanium alloys have low thermal conductivity and high chemical activity, making the cutting heat is not easy to dissipate during processing, resulting in serious thermal impact, material modification and adhesion to the tool, which greatly increases tool wear [2].

As a non-traditional high-energy beam processing technology, abrasive waterjet (AWJ) removes material by the erosion process, wherein hard abrasive particles are suspended in a high velocity of the water jet stream, which, in turn, increases the acceleration of the abrasive particles, and their 
kinetic energy impingement towards the target material, causing material removal [3]. Liao et al. [4] demonstrated that $\mathrm{AWJ}$ is a particular non-conventional machining operation that can increase the material removal rate, especially for superalloys. Also, Haghin et al. [5] claimed that AWJ can machine a various range of ductile and brittle materials, and it has excellent characteristics such as high processing efficiency, no thermal effect, and environmental benign. Moreover, AWJ cutting is superior to many other cutting technologies in processing various materials, particularly in contouring or profile cutting and in processing difficult-to-cut materials such as titanium alloys [6]. However, the cutting performance of hard materials is still far from satisfactory, which greatly limits the application [7].

It is known that the kerf quality and the processing time of AWJ cutting significantly depend on the processing parameters and strategies used, which have attracted the attention of many researchers [8]. Most of the previous studies on AWJ cutting are single cutting, and the main work done is to find the optimal cutting process parameters for different materials. Multi-pass cutting is a promising strategy for improving the AWJ cutting performance, and it represents a fertile field for more investigations, especially for the combined influence of the control parameters at consequent cutting passes on the output responses [9]. Hashish and Plessis [10] established a series of prediction equations to study the effects of standoff distance (SOD) and multi-pass strategy on the cutting depth, volume removal and specific energy. It was found that there is an optimal SOD in the water jet cutting process, and multi-pass can effectively improve the cutting quality. Wang [11] conducted experiments with alumina ceramic as the workpiece, and analyzed the process of multi-pass cutting. The research work focused on the influence of the nozzle traverse speed and direction on the cutting performance. Research shows that multi-pass cutting demonstrates distinct superiority over the single pass cutting, which provides guidance for the selection of cutting parameters. Miao et al. [12] used AISI 304 stainless steel as the workpiece, studied the quality of the cross-section with different cutting times and compared it with the cross-section after single cutting. Through experiments, they found that the optimal cutting passes are two passes and the minimum taper angle can be obtained with three passes. Xiao et al. [13] discussed the multi-pass cutting on CFRP and makes a comparison between constant and changed parameters. They found that multi-pass cutting could further reduce $53 \%$ of kerf taper and improve efficiency $13 \%$ as appropriate parameters selected without sacrifice of kerf quality. Mesalamy and Youssef [14] investigates the influence of number of cutting passes, traverse speed, and cutting direction on the quality of cutting by using Response Surface Method (RSM). The research shows that second pass is the key point to improve the surface quality.

Although numerous studies on multi-pass cutting have been conducted [15], few with variable parameters could be found in the literature, let alone on using multi-pass cutting for titanium alloys. The geometric features and dimensions that have been reported in the articles are circularity deviations and taper angle, and it is noted that SOD is the most important factor in such regards [16]. So, in the present study, a new strategy of reverse cutting with variable SOD was put forward-cut twice at the same part of the workpiece with changing the traverse direction and SOD during the second cutting. Considering whether the workpiece is cut through at one time or not, the reverse cutting was defined as reverse trimming cutting and reverse deepening cutting. It can be seen from Fig. 1 that in the second processing of reverse trimming cutting, only the edge part of the jet beam participates in the processing. The jet that is far from the nozzle has a more serious divergence. Therefore, the SOD has a great impact on the area and effect of the trimming process. In the second cutting of reverse deepening cutting, the actual SOD becomes the distance between the nozzle and 
the processing bottom surface formed in the first cutting. Therefore, it is necessary to study the influence of variable SOD on the machining quality.

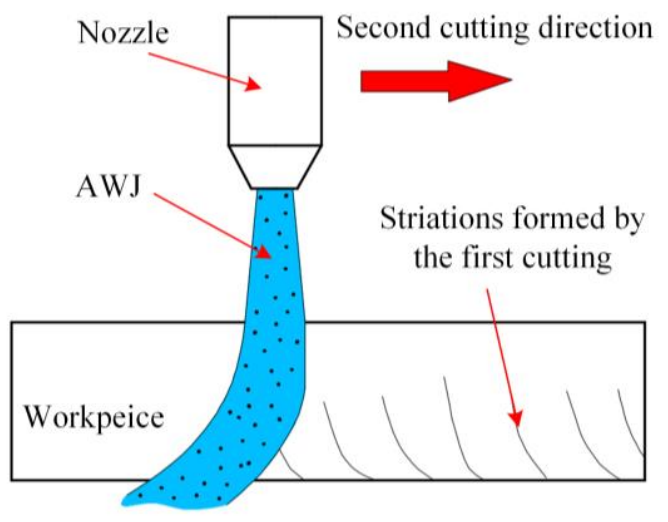

(a) Reverse trimming cutting

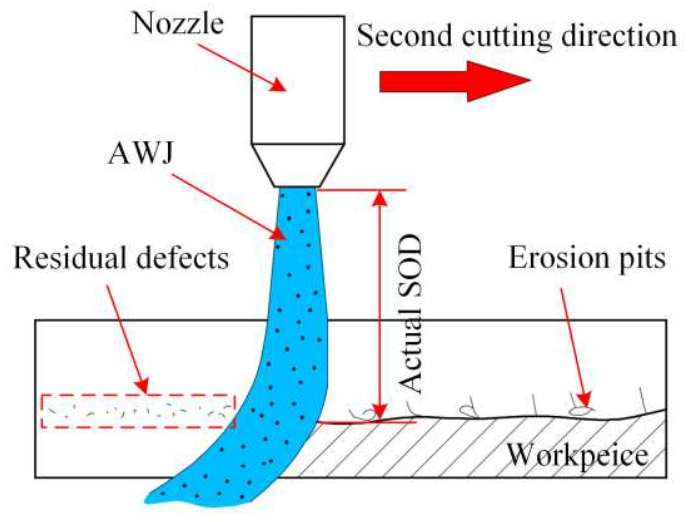

(b) Reverse deepening cutting

Fig. 1 The process of the second cutting.

The new strategy was used to investigate cutting performance on titanium alloy Ti6Al4V. At first, through the analysis of preliminary experimental data, the optimal SOD of AWJ cutting titanium alloy under single cutting condition was explored. Then, the advantages of the reverse cutting with variable SOD strategy were verified. Finally, the effects of this strategy on the cutting kerf taper and the surface quality of the cross-section were studied respectively, and the optimal parameters were obtained, which can further improve the cutting performance and reduce the cutting time at the same time. This strategy can also provide some guidance for processing other hard materials.

\section{Experimental material and methods}

\subsection{Equipment}

The experiments were performed by using a five-axis abrasive water jet machine (Model: APW2016BA-18) shown in Fig. 2, its working pressure can be adjusted steplessly with a maximum value of 420MPa. For the cutting head, a ruby nozzle with a diameter of $0.33 \mathrm{~mm}$, and a mixing tube with a diameter of $1.02 \mathrm{~mm}$ and a length of $76.2 \mathrm{~mm}$ were used in the experiments.
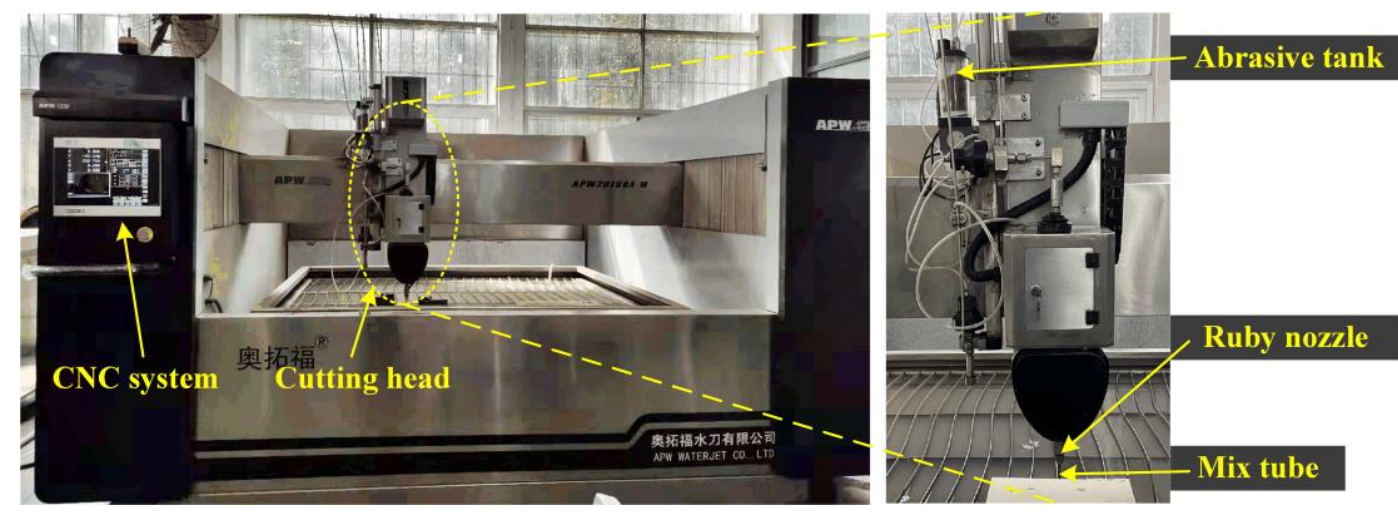

Fig. 2 Abrasive water jet machine.

The surface roughness of the machined specimen was measured on a surface test equipment (Mitutoyo SJ-210, as shown in 
Fig. 3 Surface roughness meter.

Fig. 4 3D laser scanning profiler.

) and a 3D laser scanning optical profilometers (Nanofocus uscan select, as shown in Fig. 4). The measurement range of SJ-210 for the surface height of the workpiece is $0-360 \mu \mathrm{m}$. It was used to get the $R a$ at the measuring line of the surface. Nanofocus uscan select is optical profilometers for the three-dimensional measurement and analysis of surfaces. Its resolution in the perpendicular direction is $35 \mathrm{~nm}$. It was used to obtain surface morphology and measure $S a$.

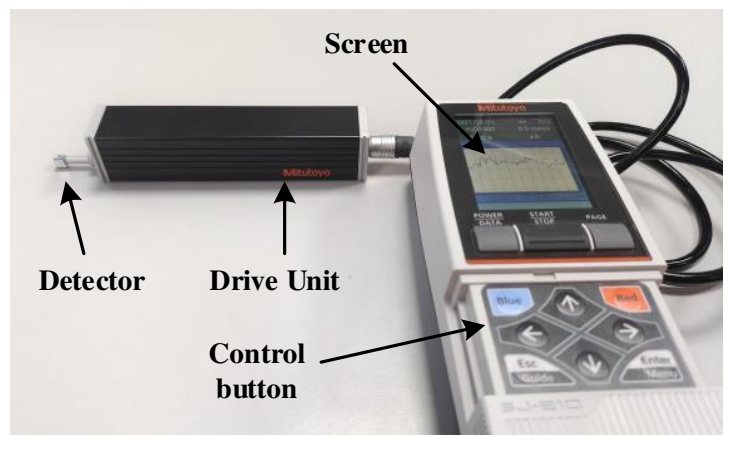

Fig. 3 Surface roughness meter.

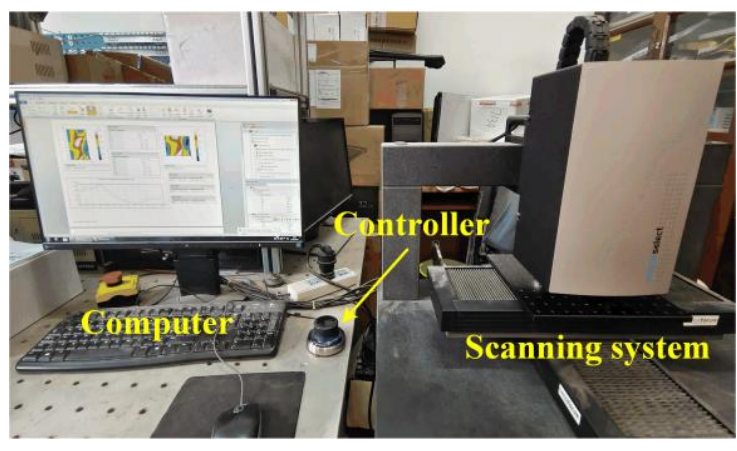

Fig. 4 3D laser scanning profiler.

\subsection{Material}

Ti6Al4V, which is a typical kind of titanium alloy, was used as the test specimen in the experiments. The physical properties of Ti6Al4V are shown in Table. 1.

Table. 1 Main physical properties of the Ti6Al4V specimen

\begin{tabular}{ccccc}
\hline $\begin{array}{c}\text { Vickers } \\
\text { hardness } \\
\left(\mathrm{H}_{\mathrm{V}}\right)\end{array}$ & $\begin{array}{c}\text { yield strength } \\
(\mathrm{MPa})\end{array}$ & $\begin{array}{c}\text { Young's modulus } \\
(\mathrm{GPa})\end{array}$ & $\begin{array}{c}\text { Density } \\
\left(\mathrm{g} / \mathrm{cm}^{3}\right)\end{array}$ & $\begin{array}{c}\text { chemical } \\
\text { composition }\end{array}$ \\
\hline \multirow{2}{*}{330} & 480 & 110 & & Ti: $90 \%$ \\
& & & 4.51 & $\mathrm{Al}: 5.5-6.8 \%$ \\
$\mathrm{~V}: 3.5-4.5 \%$
\end{tabular}

\subsection{Method}

During the AWJ cutting experiment, the nozzle was perpendicular to the surface of the workpiece and maintains a certain value of SOD. Due to the energy dissipation during AWJ machining, the kerf widths at entrance and exit are different as shown in Fig. 3b. The surface of cross-section also shows different profiles including initial zone, smooth zone, and rough zone, the range of the initial zone is very small and usually not considered [17]. The smooth zone and rough zone are produced by particles impacting at shallow and large impact angle [18]. And the rough zone has obvious striation, which can be clearly distinguished as shown in Fig. 5. 


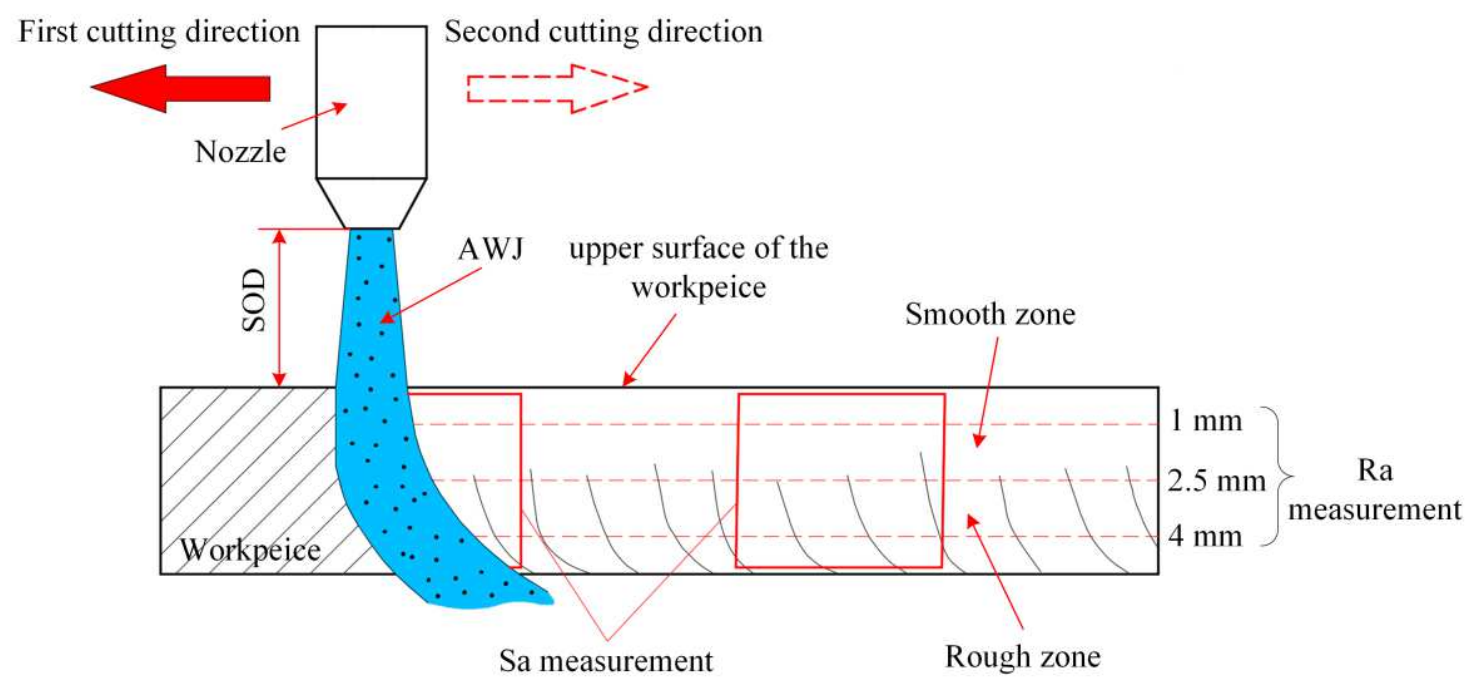

Fig. 5 The process of AWJ cutting

Since the surface of the cross-section after AWJ cutting is not uniform, it is not appropriate to simply use $R a$ to characterize the surface quality. $S a$ (Arithmetic average height of the surface) was also used to evaluate the surface quality, because $S a$ is not affected by the result error caused by the measurement position and the scanning direction in $R a$ measurement.

In this study, $S a, R a$ and kerf taper $\alpha$ (as shown in Fig. 6) were used to evaluate the machining quality. A $5 \mathrm{~mm} * 5 \mathrm{~mm}$ area on the processed cross-section was selected at an interval of $10 \mathrm{~mm}$ to be scanned, and $S a$ was then calculated by standard. Each $R a$ was measured three times at positions of $1 \mathrm{~mm}, 2.5 \mathrm{~mm}$, and $4 \mathrm{~mm}$ down from the upper surface of the workpiece (as shown in Fig. 5). The entrance width and exit width are measured on three different locations of the kerf. The kerf taper $\alpha$ can be described by the following equation:

$$
\alpha=\arctan \left(\frac{W_{a}-W_{b}}{2 h}\right)
$$

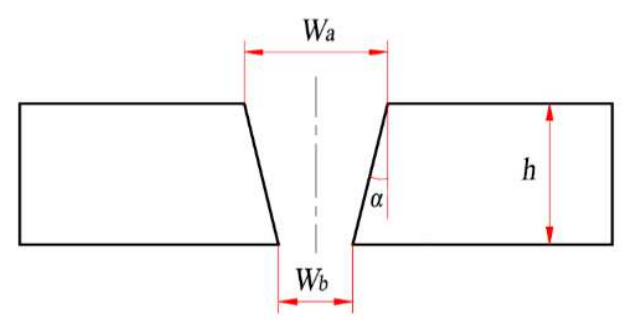

Fig. 6 Kerf properties after AWJ cutting

This study mainly focuses on the influence of changing the SOD of the second cutting on the cutting performance under different traverse speed. The constant parameters are shown in Table. 2.

Table. 2 Value of constant parameters.

\begin{tabular}{ccccc}
\hline $\begin{array}{c}\text { Pressure } \\
(\mathrm{MPa})\end{array}$ & $\begin{array}{c}\text { Impact angle } \\
\left({ }^{\circ}\right)\end{array}$ & $\begin{array}{c}\text { Type of } \\
\text { abrasive }\end{array}$ & Abrasive size & $\begin{array}{c}\text { Abrasive flow rate } \\
(\mathrm{g} / \mathrm{min})\end{array}$ \\
\hline 320 & 90 & Garnet & $80 \#$ & 480 \\
\hline
\end{tabular}

All experiments are conducted twice and the results are an average of these measurements. The error bars of each data in the paper are the standard error of the measured value. 


\section{Preliminary tests}

\subsection{The range of traverse speed}

Preliminary tests were performed in advance to get the appropriate parameters, which were the working pressure, the traverse speed, and the SOD.

The parameters in Table. 2 were used to cut a piece of Ti6Al4V with a thickness of $10 \mathrm{~mm}$, the cutting depth of the traverse speed in the range of $400-1600 \mathrm{~mm} / \mathrm{min}$ is determined, as shown in Fig. 7. This curve serves as an important reference for the selection of the later movement speed in the study of variable SOD.

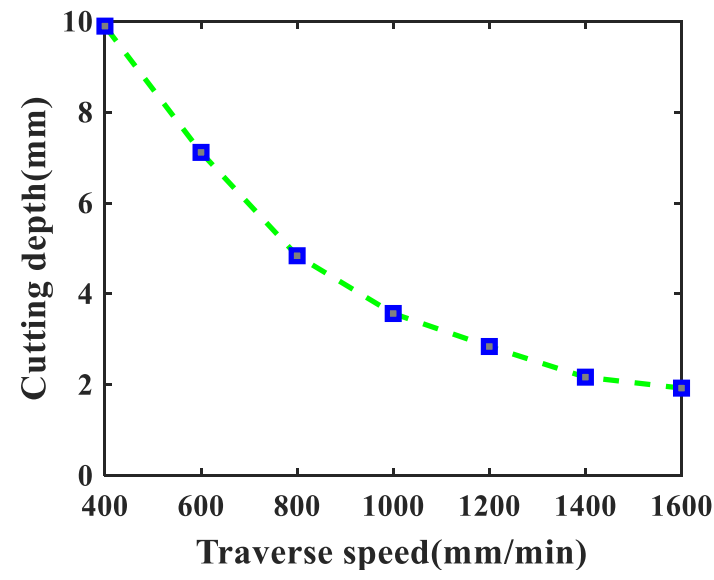

Fig. 7 Cutting depth at different traverse speeds

It can be seen that the cutting depth decreases with the increase of traverse velocity, but the decreasing trend becomes slow gradually.

\subsection{The optimal SOD}

It is known that there exists an optimal value of SOD, which depends on the machining conditions [19]. Therefore, it is important to find the optimal SOD for single cutting under the condition. According to the curve in Fig. 7, when the traverse speed is $800 \mathrm{~mm} / \mathrm{min}$, the cutting depth is about $5 \mathrm{~mm}$. The workpieces with a width of $50 \mathrm{~mm}$ and a thickness of $5 \mathrm{~mm}$ were used for the single cutting tests, and the scheme is shown in Table. 3

Table. 3 Single cutting tests parameter

\begin{tabular}{cc}
\hline Traverse speed(mm/min) & SOD $(\mathrm{mm})$ \\
\hline $400 / 600 / 800$ & $2 / 4 / 6 / 8 / 10$ \\
\hline
\end{tabular}

\subsubsection{Surface quality}

\section{a) Macroscopic analysis of the cross-section}

Nine cross-sections under the condition of the SOD were $2 \mathrm{~mm}, 6 \mathrm{~mm}$ and $10 \mathrm{~mm}$ at three traverse speeds were used to analyze the surface characteristics. 

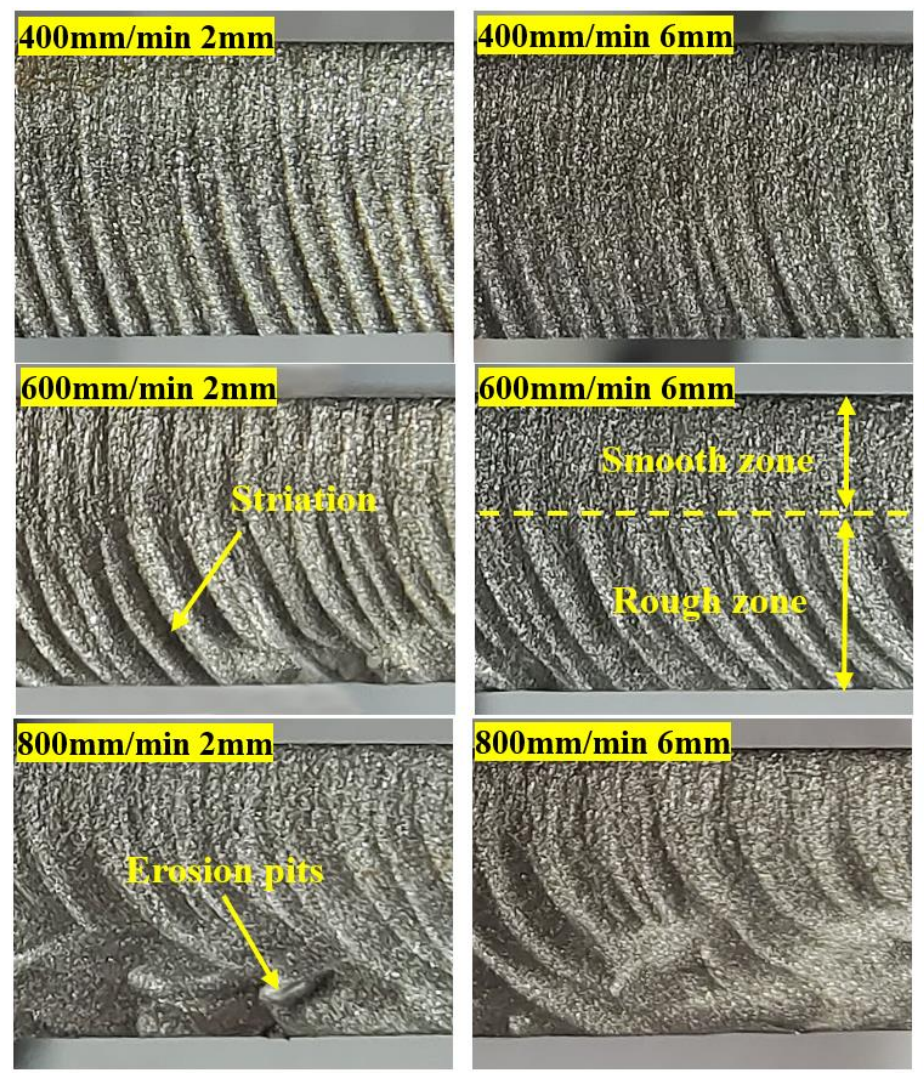
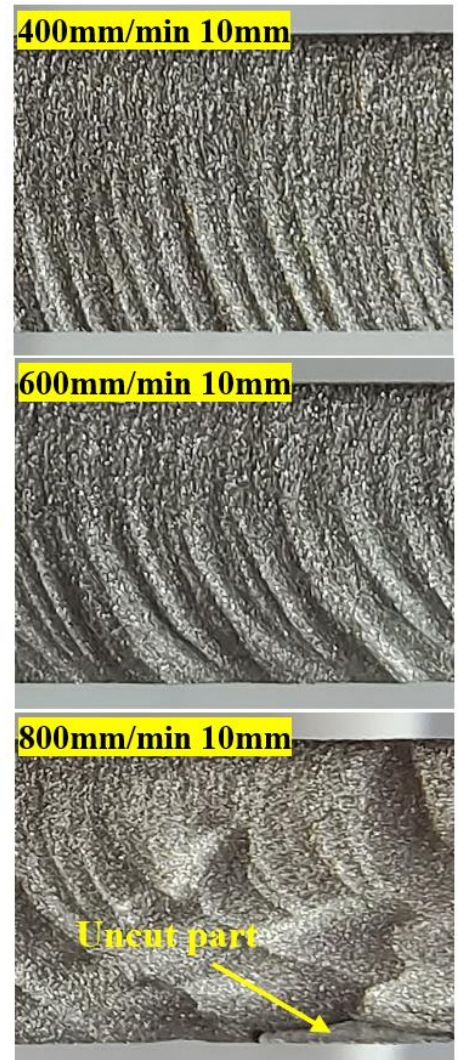

Fig. 8 Surface characteristics of cross-section after single cutting

As can be seen from Fig. 8, the cross-section of the workpiece after a single cutting presents obvious partitions. With the increase of traverse speed, the smooth zone decreases and the surface deteriorates gradually. When the traverse speed is $800 \mathrm{~mm} / \mathrm{min}$, erosion pits and uncut parts appear on the surface, leading to a sharp decline in surface quality. Comparing the cross-sections of different SOD, it can be seen that the surface quality is worse when the SOD is too small or too large. Therefore, for a specific workpiece, there must be an optimal SOD to obtain the best surface quality. In order to obtain the optimal SOD, the surfaces were quantitatively analyzed by using $R a$ and $S a$ to characterize the cross-sections quality.

\section{b) Surface roughness analysis}

In order to acquire the optimal SOD for cutting titanium alloy under the certain working conditions, surface test and 3D profilometers were used to obtain the data representing the surface quality of the cross-section. 


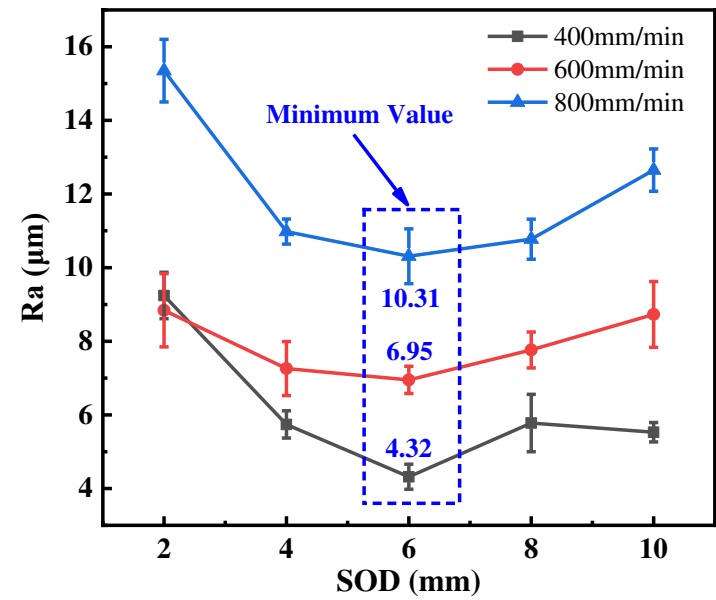

(a) $R a$ in different parameters

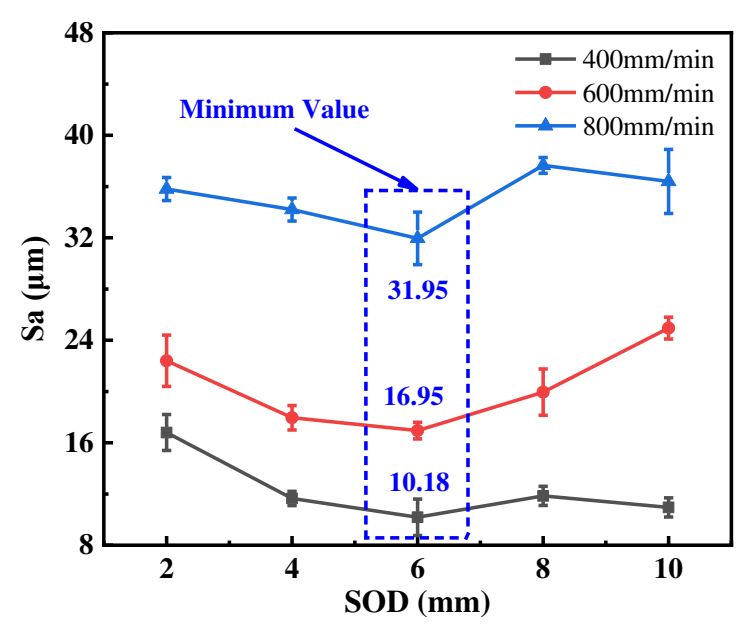

(b) $\mathrm{Sa}$ in different parameters

Fig. 9 Cross-section roughness of single cutting

It can be seen from Fig. 9 that at three traverse speeds, when the SOD is about $6 \mathrm{~mm}$, the $R a$ and $S a$ of the cross-section are minimum. Moreover, with the traverse speed increasing from $400 \mathrm{~mm} / \mathrm{min}$ to $800 \mathrm{~mm} / \mathrm{min}$, the minimum value of $R a$ increases from $4.32 \mu \mathrm{m}$ to $10.31 \mu \mathrm{m}$, and the minimum value of $S a$ increases from $10.18 \mu \mathrm{m}$ to $31.95 \mu \mathrm{m}$, both of which increase about three times. It can be observed from Fig. 8 that when the SOD is $6 \mathrm{~mm}$, the smooth area of the cross-section is larger than that when the SOD is $2 \mathrm{~mm}$ or $10 \mathrm{~mm}$. The reason for this result is that SOD has an important correlation with the divergence and energy distribution of AWJ. When the SOD is too small, the acceleration of the abrasive is insufficient, resulting in a weaker removal ability of the AWJ. When the SOD is too large, the energy attenuation due to the friction between the AWJ beam and the air will also weaken the removal ability of the AWJ. When the removal ability of AWJ is weak, it will enlarge the rough area of the cross-section, which will increase the roughness of the cross-section and reduce the surface quality.

It is of great interest to find that the values of $R a$ and $S a$ increase with the a higher traverse speed, especially when the traverse speed is $800 \mathrm{~m} / \mathrm{min}$. Combining the cross-section in Fig. 8 to analyze the reason is that when the traverse speed is small, the AWJ beam deflection during the cutting process is not obvious, so most of the water and abrasive are ejected from the exit, which can obtain a more regular and flatter striations of cross-section. With the increase of traverse speed, the speed reaches the critical value that cannot cut through the workpiece. The deflection of AWJ is severe, and some random direction reflected jets are generated, which will damage the machined surface, resulting in irregular erosion pits in the cross-section, so the surface quality drops sharply.

\subsection{2 kerf taper}

A microscope was used to measure the width of the kerf entrance and exit, and then calculate the kerf taper. It can be seen from Fig. 10a that the kerf taper increases as the SOD increases at different traverse speeds. For example, at a traverse speed of $600 \mathrm{~mm} / \mathrm{min}$, the kerf taper increases from $3.21^{\circ}$ to $4.96^{\circ}$ as the SOD increasing form $2 \mathrm{~mm}$ to $10 \mathrm{~mm}$, a significant increase. 


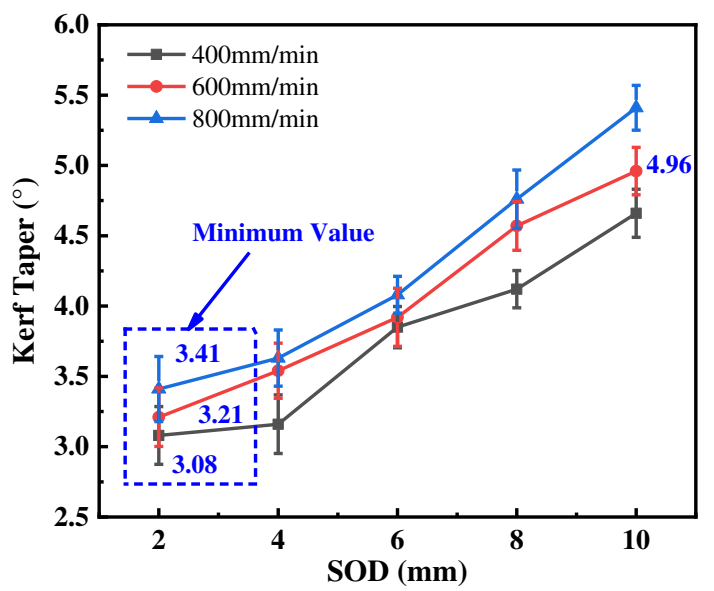

(a) Kerf taper

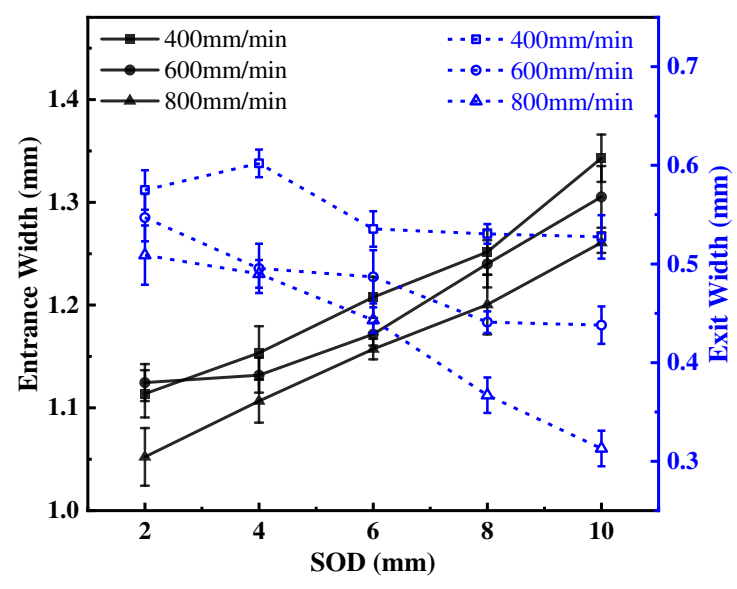

(b) Kerf width

Fig. 10 Kerf width and taper of single cutting

According to the analysis in Fig. 10b, when the SOD is small, the jet divergence is small and the acceleration process of abrasive particles is short, resulting in weak cutting ability, which forms a narrow kerf entrance width. With the increase of the SOD, the jet diverges severely and the acceleration of the abrasive particles is also more sufficient, resulting in stronger removal ability, which forms a wider kerf entrance width. During the cutting process, due to the energy consumption of the AWJ, the exit width is less than the entrance width. As SOD increases, the greater the dispersion and the consumption of AWJ energy. As a result, the high-speed abrasive particles participating in the cutting process gradually decrease with the depth of the cutting, so the exit width becomes narrower.

Moreover, when the SOD is $2 \mathrm{~mm}$, the minimum value of kerf taper increases from $3.08^{\circ}$ to $3.41^{\circ}$ with the traverse speed increasing from $400 \mathrm{~mm} / \mathrm{min}$ to $800 \mathrm{~mm} / \mathrm{min}$. Obviously, the increase of the traverse speed makes the AWJ cutting time shorter, which will inevitably lead to smaller jet energy and weaker cutting ability. Therefore, the faster the nozzle traverse speed, the smaller the width of the formed entrance and exit.

Taking surface quality as the first consideration, $6 \mathrm{~mm}$ was chosen as the SOD for the first cutting. Since the rules obtained at the three speeds are the same, $600 \mathrm{~mm} / \mathrm{min}$ is chosen as the traverse speed for subsequent experiments. Then the traverse speed of $1200 \mathrm{~mm} / \mathrm{min}$, which can keep the same processing time with the single cutting, was selected as the comparison. The improvement of reverse trimming cutting and reverse deepening cutting on machining quality was studied respectively. Experimental parameters are shown in the Table. 4.

Table. 4 Experiment parameter

\begin{tabular}{ccc}
\hline Strategy & Reverse trimming cutting & $\begin{array}{c}\text { Reverse deepening } \\
\text { cutting }\end{array}$ \\
\hline Traverse speed $(\mathrm{mm} / \mathrm{min})$ & 600 & 1200 \\
SOD in first cutting $(\mathrm{mm})$ & 6 & 6 \\
SOD in second cutting $(\mathrm{mm})$ & $2 / 4 / 6 / 8 / 10$ & $2 / 4 / 6 / 8 / 10$ \\
\hline
\end{tabular}

\section{Results and discussion}


In order to clearly show the effects of the new machining strategy, the surface characteristics of the cross-sections under different machining strategies were analyzed at first. Then the optimal process parameters were obtained by a quantitative analysis of the surface roughness of the cross-section.

\subsection{Macroscopic analysis of different cutting strategy}

Specimens with better surface quality after processing by the new strategy and the specimens after cutting without changing the SOD in second cutting were used for comparative analysis. The processed cross-sections are shown in Fig. 11. Use the three-dimensional profiler to scan and reconstruct the processed cross-sections, the surface morphology of the cross-sections can be obtained, as shown in Fig. 12.
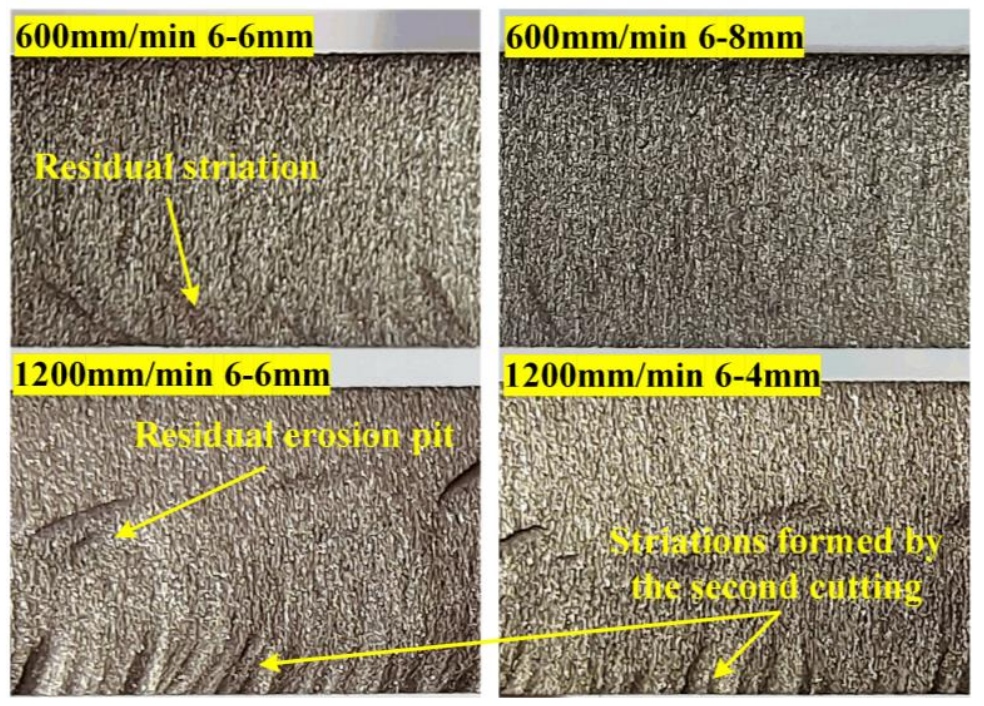

Fig. 11 Cross-sections after reverse cutting

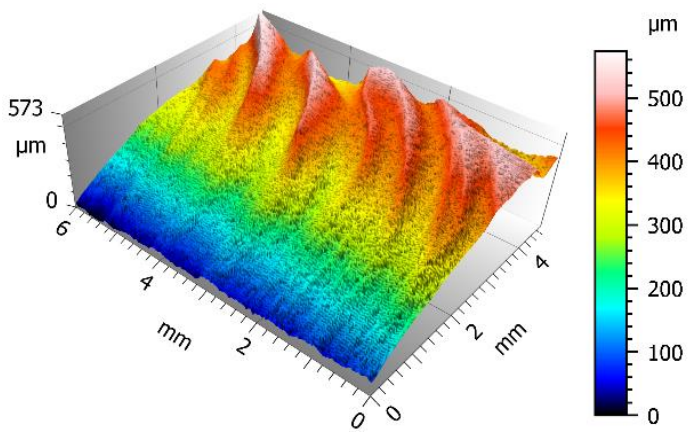

(a) Control group-single cutting

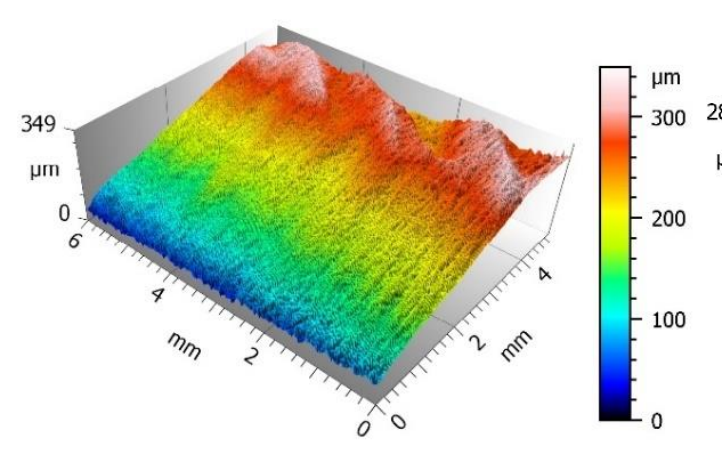

(b) $600 \mathrm{~mm} / \mathrm{min} 6-6 \mathrm{~mm}$

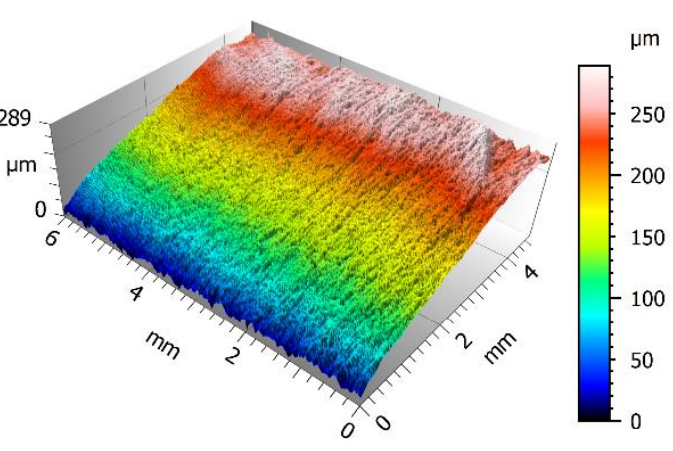

(c) $600 \mathrm{~mm} / \mathrm{min} 6-8 \mathrm{~mm}$ 


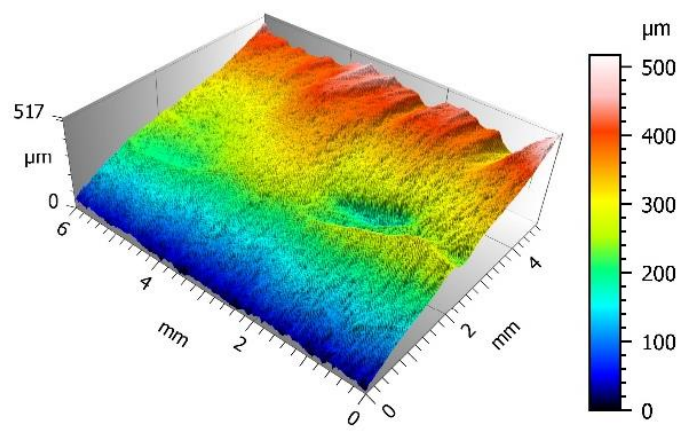

(d) $1200 \mathrm{~mm} / \mathrm{min} 6-6 \mathrm{~mm}$

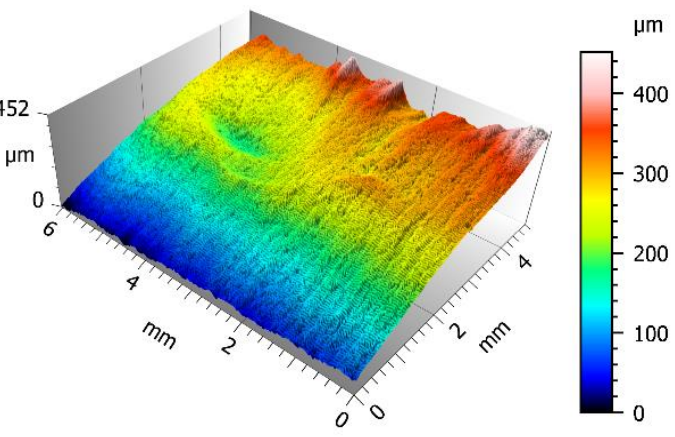

(e) $1200 \mathrm{~mm} / \mathrm{min} 6-4 \mathrm{~mm}$

Fig. 12 Surface morphology of the cross-sections

Taking both Fig. 11 and Fig. 12 into consideration, it can be found that when the traverse speed is the same as the single cutting, the processing method is reverse trimming cutting. Compared with the surface after a single cutting in Fig. 8, the surface processed in the way is quite flat, and the rough area on the surface almost disappears, except for a small number of residual striations near the exit of the kerf, and the number and size of striations are reduced exponentially.

From Fig. 12, it can be seen that the height difference between the peaks and valleys of striation are reduced from about $200 \mu \mathrm{m}$ for single cutting to about $80 \mu \mathrm{m}$ for new machining strategy. In addition, the inclination of the cross-section also decreased, with the difference between the exit and the entrance decreasing from $573 \mu \mathrm{m}$ to $349 \mu \mathrm{m}$. As shown in Fig. 12c, the number and size of residual striations on the cross-section obtained by appropriately increasing the SOD before the second cutting are fewer and the surface is smoother.

To keep the processing time the same as the single cutting, a traverse speed of $1200 \mathrm{~mm} / \mathrm{min}$ is used. In this case, because the traverse speed is fast, the workpiece is not cut through in the first processing, and the processing method is the reverse deepening cutting. In this way, the residual striations on the processed surface is also greatly reduced relative to a single cutting. However, the non-through cutting produces the pocket at the bottom of the kerf due to unordered jet upward deflection [16]. Hence, the kerf produced at first-pass cutting occasionally presented different shapes that could influence the performance at second pass such as the irregular pits randomly occurring at kerf wall. After the second cutting, the erosion pits formed in the first cutting was trimmed to a certain extent. Compared with the height of striations on the surface after a single cutting, the erosion pits depth is smaller, the surface quality is still better than that of a single cutting, and the height difference between the entrance and exit is also somewhat reduced. Meanwhile, comparing Fig. 12d and Fig. 12e, it can be seen that when the SOD reaches the appropriate value for the second cutting of reverse deepening cutting, the removal effect of the defects generated in the first processing is significant, so the quality of the cross-section after the cutting is better.

\subsection{Research on surface quality}

In order to quantitatively demonstrate the specific improvement of the reverse cutting with variable SOD strategy on the surface quality after machining, the surface processed by different strategies were measured, and the results are shown in Fig. 13. For the two different types of processing methods in the new strategy, the parameters for obtaining higher quality are different, and the two methods need to be analyzed separately. 


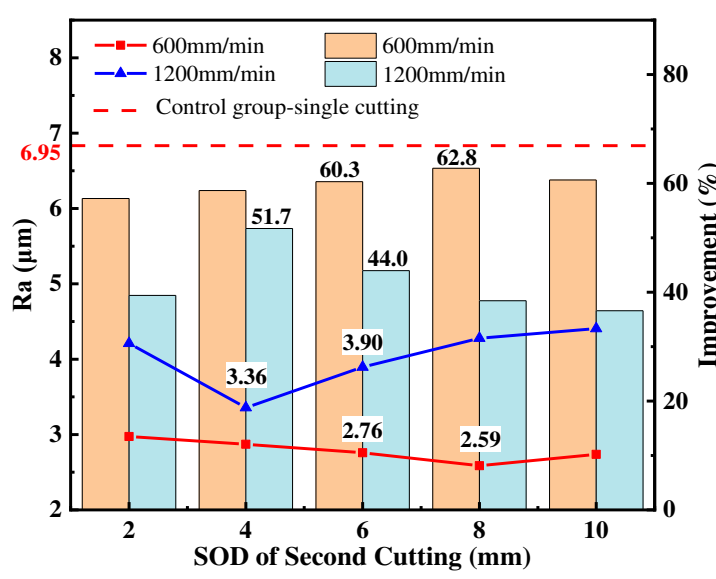

(a) $R a$ in different strategy

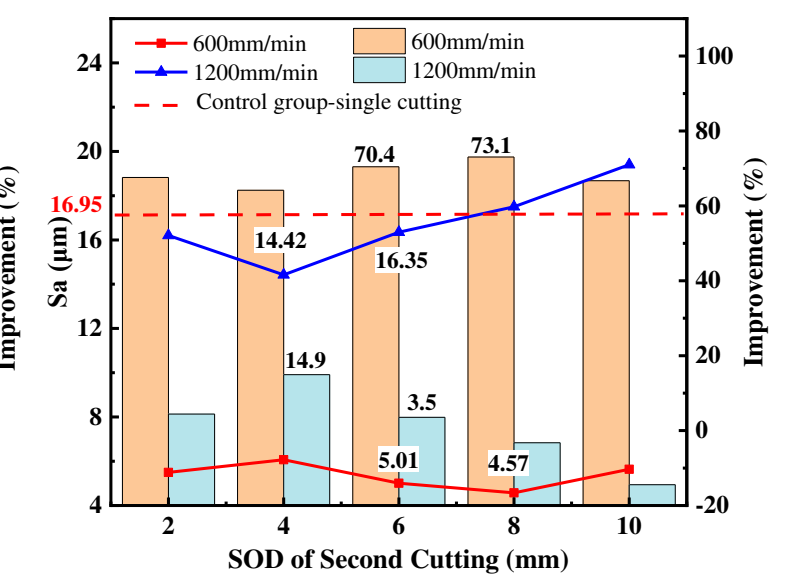

(b) $S a$ in different strategy

Fig. 13 Surface quality of cross-section

Fig. 13a shows that $R a$ values of the surface cut by the reverse deepening cutting method at the traverse speed of $1200 \mathrm{~mm} / \mathrm{min}$ are between $3.3-4.5 \mu \mathrm{m}$. According to the standard definition, when $R a$ is $3.2 \mu \mathrm{m}$, the surface features are slight processing traces. However, it can be clearly seen from Fig. 12 that there are surface defects, which are not consistent with the situation represented by $R a$. So, the center line average value parameter $R a$ is limited in characterizing the surface quality of the uneven surface. When the rough area is outside the measurement position, the surface quality characterized by the $R a$ mismatch the actual. This problem does not exist in the $S a$ measurement, because the measurement points of $S a$ are evenly distributed throughout the measurement area, so it is more accurate to use $S a$ to characterize the quality of uneven surfaces.

\section{a) The reverse trimming cutting}

For reverse trimming cutting, the surface quality of the cross-section is directly related to the amount of trimming. Different jet divergence degrees get different trimming amounts, and the jet divergence degree is positively correlated with the SOD.

It can be seen from the Fig. 13 that compared to a single cutting with a traverse speed of $600 \mathrm{~mm} / \mathrm{min}$, when the SOD of the second cutting is $8 \mathrm{~mm}$, the reverse trimming cutting at the same traverse speed has the best effect on improving the surface quality of the cross-section. The improvements of $R a$ and $S a$ are $62.8 \%$ and $73.1 \%$. At this time, the $R a$ and $S a$ of the cross-section are 2.59 and $4.57 \mu \mathrm{m}$. However, if the SOD is not changed during the reverse trimming process, $R a$ and $\mathrm{Sa}$ only increase by $60.3 \%, 70.4 \%$.

The reason for the above results is that when the jet divergence is small, the contact area between the jet and the workpiece is small, and the surface trimming degree is low. As the SOD increases, the jet beam diverges significantly, and the trimming area is wider. When the SOD is too large, the jet diverges severely and damages the existing surface, which causing the surface quality to degrade again.

\section{b) The reverse deepening cutting}

Reverse deepening cutting means to increase the cutting depth through the second reverse cutting, so as to achieve the purpose of cutting through the workpiece. When the current traverse speed cannot cut through the workpiece at one time, the influence of the SOD of the second cutting on the cross-section quality was studied.

The result of measuring the cross-section is shown in Fig. 13. It can be seen that $R a$ and $S a$ obtain the minimum value when the SOD of the second cutting is $4 \mathrm{~mm}$. When the SOD of the 
second cutting is further increased, the surface quality will deteriorate.

While maintaining the same time as the single cutting with a traverse speed of $600 \mathrm{~mm} / \mathrm{min}$, the reverse deepening cutting with a traverse speed of $1200 \mathrm{~mm} / \mathrm{min}$ is used for processing. Compared to the single cutting, when the SOD of the second cutting is $4 \mathrm{~mm}, R a$ and $S a$ obtain the best enhancement effects, which are $51.7 \%$ and $14.9 \%$ respectively. At this time, the $R a$ and $S a$ of the cross-section are 3.36 and $14.42 \mu \mathrm{m}$. However, if the SOD of the reverse deepening cutting is kept unchanged, $R a$ and $S a$ only increase by $44.0 \%$ and $3.5 \%$.

\subsection{Research on kerf taper}

As is shown in Fig. 14, by appropriately reducing the SOD in the second cutting, higher cutting capacity can be obtained. the kerf taper gets the minimum value when the SOD of the second cutting is $2-4 \mathrm{~mm}$. As the SOD increase, the kerf taper increases gradually.

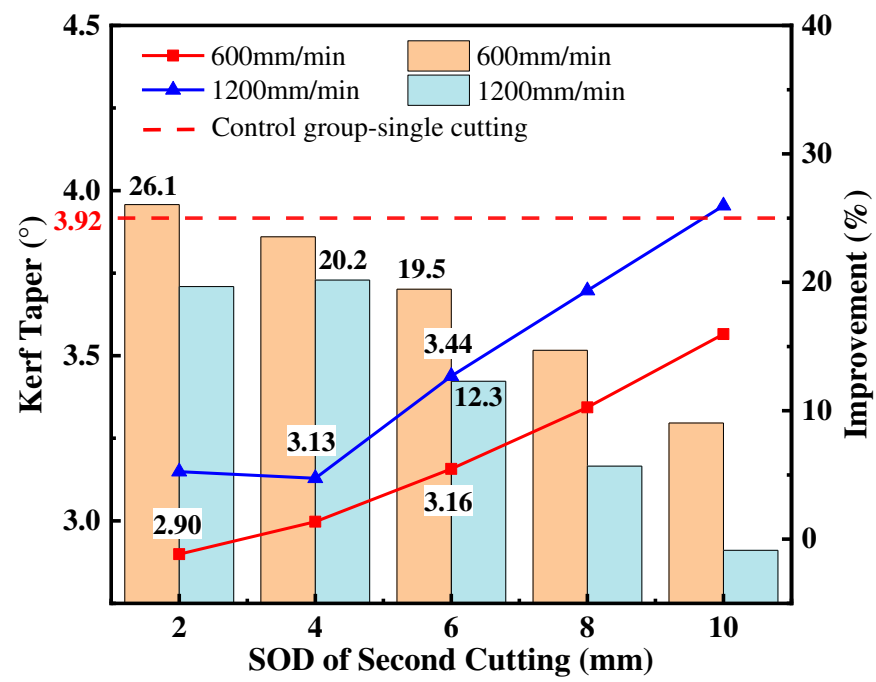

Fig. 14 Improvement of kerf taper

Compared to a single cutting with a traverse speed of $600 \mathrm{~mm} / \mathrm{min}$, when the SOD of the second cutting is $2-4 \mathrm{~mm}$, kerf taper $\alpha$ after reverse trimming cutting and reverse deepening cutting obtain the best enhancement effects, which are $26.1 \%$ and $20.2 \%$. At this time, the kerf taper $\alpha$ are $2.90^{\circ}$ and $3.13^{\circ}$. However, if the SOD is not changed during the second cutting process, kerf taper $\alpha$ only decrease by $19.5 \%$ and $12.3 \%$ 。

\section{Conclusion}

In this study, a new strategy of reverse cutting with variable SOD has been used to improve the cutting quality aluminum alloy machined by AWJ. As the SOD changes with the progress of the reverse processing, the strategy of adjusting the SOD in the reverse cutting represents good results for kerf quality compared to a single cutting.

(1) For processing methods with uneven cross-sections after processing, the commonly used center line average value parameter $R a$ is not accurate enough to characterize the actual surface quality. The combination of Sa and RA can make up for the deficiency of RA in the characterization of uneven surfaces.

(2) In the reverse trimming process, in order to obtain better cross-section quality, it is necessary to appropriately increase the SOD during the second cutting. In order to reduce the kerf 
taper, the SOD needs to be reduced during the second cutting. Under the condition of a traverse speed of $600 \mathrm{~mm} / \mathrm{min}$, when the SOD is $8 \mathrm{~mm}$ for the second cutting, the $R a$ and $S a$ when the optimal surface quality is obtained are $2.59 \mu \mathrm{m}$ and $4.57 \mu \mathrm{m}$, respectively. Compared to a single cutting at the same speed, the improvements of $R a$ and $S a$ are $62.8 \%$ and $73.1 \%$. When the SOD of the second cutting is $2 \mathrm{~mm}$, the minimum kerf taper obtained is $2.90^{\circ}$, reducing $26.1 \%$ compared to a single cutting.

(3) For reverse deepening cutting, in order to obtain the best kerf quality (including surface quality and kerf taper), it is necessary to appropriately reduce the SOD. When the traverse speed is $1200 \mathrm{~mm} / \mathrm{min}$, its cutting process maintains the same cutting efficiency as a single cutting of $600 \mathrm{~mm} / \mathrm{min}$. At this time, when the SOD of second cutting is $4 \mathrm{~mm}, R a, S a$ and $\alpha$ all achieve the minimum value. They are 3.36 and $14.42 \mu \mathrm{m}$, and $3.13^{\circ}$. The improvement are $51.7 \%, 14.9 \%$ and $20.2 \%$, respectively.

\section{Funding}

This research is financially supported by the National Natural Science Foundation of China (Nos.52175245 and 51805188), and the Natural Science Foundation of Hubei (No.2021CFB462).

\section{Conflicts of interest}

The authors declare that they have no known competing financial interests or personal relationships that could have appeared to influence the work reported in this paper.

\section{Availability of data and material}

All the data sets supporting the results are included within the article.

\section{Code availability}

Not applicable.

\section{Ethics approval}

Not applicable.

\section{Consent to participate}

Not applicable.

\section{Consent for publication}

All authors agree to transfer copyright of this article to the Publisher.

\section{Authors' contributions}

Jie Xiong: Conceptualization, Investigation, and Writing. Liang Wan: Methodology and Formal Analysis. Yi'nan Qian: Data collecting and editing. Shuo Sun: Formal Analysis. Deng Li: Project administration, and Writing-review and editing. Shijing Wu: Supervision. 


\section{References}

[1] Wang, F. , Yang, K. , Xiao, N. , Li, L. , Guo, C., 2021. Quantitative analysis on the surface topography of Ti-6Al-4V machined by abrasive suspension jet. Int. J. Adv. Manuf. Technol., 112(1-2), 1-10.

[2] Liao, Z., Sanchez, I., Xu, D., Axinte, D., Augustinavicius, G., Wretland, A., 2020. Dual-processing by abrasive waterjet machining-A method for machining and surface modification of nickel-based superalloy. J. Mater. Process. Technol., 285, 116768.

[3] Gnanavelbabu, A., Saravanan, P., Rajkumar, K., Karthikeyan, S., 2018. Experimental investigations on multiple responses in abrasive waterjet machining of Ti-6Al-4V alloy. Mater. Today: Proc., 5(5), 13413-13421.

[4] Liao, Z., Xu, D., Luna, G. G., Axinte, D., Augustinavicius, G., Sarasua, J. A., Wretland, A., 2021. Influence of surface integrity induced by multiple machining processes upon the fatigue performance of a nickel based superalloy. J. Mater. Process. Technol., 298, 117313.

[5] Haghbin, N., Spelt, J. K., Papini, M., 2015. Abrasive waterjet micro-machining of channels in metals: Model to predict high aspect-ratio channel profiles for submerged and unsubmerged machining. J. Mater. Process. Technol., 222, 399-409.

[6] Feng, L., Liu, G. R., Li, Z., Dong, X., Du, M., 2019. Study on the effects of abrasive particle shape on the cutting performance of Ti-6Al-4V materials based on the SPH method. Int. J. Adv. Manuf. Technol., 101(9), 3167-3182.

[7] Natarajan, Y., Murugesan, P. K., Mohan, M., Khan, S. A. L. A., 2020. Abrasive Water Jet Machining process: A state of art of review. J. Manuf. Process., 49, 271-322.

[8] Liu, D., Nguyen, T., Wang, J., Huang, C., 2020. Mechanisms of enhancing the machining performance in micro abrasive waterjet drilling of hard and brittle materials by vibration assistance. Int. J. Mach. Tools . Manuf., 151, 103528.

[9] Khalilpourazari, S., Khalilpourazary, S., 2018. Optimization of production time in the multi-pass milling process via a Robust Grey Wolf Optimizer. Neural Comput. Appl., 29(12), 1321-1336.

[10] Hashish, M., Du Plessis, M. P., 1979. Prediction equations relating high velocity jet cutting performance to stand off distance and multipasses. J. Eng. Ind. 101(3), 311-318.

[11] Wang, J., 2004. Techniques for enhancing the cutting performance of abrasive waterjets. Key Eng. Mater., 257, 521-526. Trans. Tech. Publications Ltd.

[12] Miao, X., Qiang, Z., Wu, M., Song, L., Ye, F., 2018. The optimal cutting times of multipass abrasive water jet cutting. Int. J. Adv. Manuf. Technol., 97(5-8), 1779-1786.

[13] Xiao, S., Wang, P., Gao, H., \& Soulat, D., 2019. A study of abrasive waterjet multi-pass cutting on kerf quality of carbon fiber-reinforced plastics. Int. J.Adv. Manuf. Technol., 105(11), 4527-4537.

[14] Mohamad, W. N. F., Kasim, M. S., Norazlina, M. Y., Hafiz, M. S. A., Izamshah, R., Mohamed, S. B., 2020. Effect of standoff distance on the kerf characteristic during abrasive water jet machining. Results Eng, 6, 100101.

[15] Nair, A., Kumanan, S., Shanavas, K. P. (2021). Heuristic optimization techniques in abrasive water jet hole making-A case study. Mater. Today: Proc. (In Press)

[16] Nair, A., Kumanan, S., 2017. Multi-performance optimization of abrasive water jet machining 
of Inconel 617 using WPCA. Mater. Manuf. Process., 32(6), 693-699

[17] Chen, F. L., Siores, E., 2003. The effect of cutting jet variation on surface striation formation in abrasive water jet cutting. J. Mater. Process. Technol., 135(1), 1-5.

[18] Zhao, W., Guo, C., 2014. Topography and microstructure of the cutting surface machined with abrasive waterjet. Int. J.Adv. Manuf. Technol., 73(5-8), 941-947.

[19] El Mesalamy, A. S., Youssef, A., 2020. Enhancement of cutting quality of abrasive waterjet by using multipass cutting strategy. J. Manuf. Process., 60, 530-543. 